Article

\title{
Characterizations of Positive Operator-Monotone Functions and Monotone Riemannian Metrics via Borel Measures
}

\author{
Pattrawut Chansangiam ${ }^{1, * \mathbb{D}}$ and Sorin V. Sabau ${ }^{2} \mathbb{D}$ \\ 1 Department of Mathematics, Faculty of Science, King Mongkut's Institute of Technology Ladkrabang, \\ Bangkok 10520, Thailand \\ 2 Department of Biology, Faculty of Biological Sciences, Tokai University, 5-1-1-1 Minamisawa, Minamiku, \\ Sapporo 005-8601, Japan; sorin@tokai.ac.jp \\ * Correspondence: pattrawut.ch@kmitl.ac.th; Tel.: +66-9352-666-00
}

Received: 18 November 2019; Accepted: 27 November 2019; Published: 2 December 2019

check for updates

\begin{abstract}
We show that there is a one-to-one correspondence between positive operator-monotone functions on the positive reals, monotone Riemannian metrics, and finite positive Borel measures on the unit interval. This correspondence appears as an integral representation of weighted harmonic means with respect to that measure on the unit interval. We also investigate the normalized/symmetric conditions for operator-monotone functions. These conditions turn out to characterize monotone metrics and Morozowa-Chentsov functions as well. Concrete integral representations of such functions related to well-known monotone metrics are also provided. Moreover, we use this integral representation to decompose positive operator-monotone functions. Such decomposition gives rise to a decomposition of the associated monotone metric.
\end{abstract}

Keywords: operator-monotone function; monotone Riemannian metric; Morozowa-Chentsov function; Borel measure; density matrix

MSC: 15A63; 28A25; 53B21; 81P45; 94A17

\section{Introduction}

This paper is motivated from functional analysis aspects in quantum statistical mechanics. In classical statistics, the (Fisher) information is a measurement of the amount of information that an observable random variable conveys about an unknown parameter of its distribution. The quantum Fisher information in quantum statistics is an analogous concept to the classical one; see e.g., [1]. Recall that a physical observable of a quantum mechanical system is represented by a self-adjoint operator $A$ acting on a Hilbert space $(\mathcal{H},\langle\cdot, \cdot\rangle)$. The state of the physical system is often modeled by a unit vector $x$ in $\mathcal{H}$. In this case, the expectation of $A$ in that state is given by the inner product $\langle A x, x\rangle$. If $\operatorname{dim} \mathcal{H}<\infty$, the states (i.e., the expectation of the states) can be realized as $\operatorname{Tr}(D A)$ where $D$ is the density matrix associated with the state. In order for a difficult-to-measure observable to measure a conserved quantity, Wigner and Yanase [2] proposed the so-called skew information defined by

$$
I_{D}(A)=-\frac{1}{2} \operatorname{Tr}\left(\left[D^{\frac{1}{2}}, A\right]^{\frac{1}{2}}\right),
$$

here, $[\cdot, \cdot]$ is the commutator. Dyson introduced other measures of quantum Fisher information, namely,

$$
I_{D}(\beta, A)=-\frac{1}{2} \operatorname{Tr}\left(\left[D^{\beta}, A\right] \cdot\left[D^{1-\beta}, A\right]\right)
$$


with parameter $\beta \in(0,1)$, known as the Wigner-Yanase-Dyson skew information; see more information in [3]. Chentsov [4] proved that the Fisher information is a Riemannian metric. Morozowa and Chentsov [5] extended the analysis of quantum system by replacing Riemannian metrics with a monotone metric associated to each invertible density matrix. A monotone metric is a positive-definite sesquilinear forms $K_{D}$ defined on the tangent space of a quantum system, where $D$ is an invertible density matrix. The works [5-7] show that every monotone Riemannian metric is associated to an operator-monotone function $f:(0, \infty) \rightarrow(0, \infty)$. Hence, the theory of positive operator-monotone functions plays an important role in quantum information theory.

Many physicists and mathematicians have made contributions to this theory; see, e.g., [7-12]. Certain integral representations of operator-monotone increasing/decreasing functions are used to obtain the formulas of Morozowa-Chentsov functions associated with certain Wigner-Yanase-Dyson metrics; see $[9,13,14]$. Applications of positive operator-monotone functions and monotone metrics also arise in other areas of physics: entropy (e.g., [15]), quantum entanglement ([16]), uncertainty relations ([17]), electrical network synthesis ([18]), and condensed matter physics ([19]).

Recall that a continuous function $f:(0, \infty) \rightarrow(0, \infty)$ is said to be operator-monotone for all invertible positive operators $A$ and $B$, we have

$$
A \leqslant B \Longrightarrow f(A) \leqslant f(B),
$$

where $f(A)$ is the functional calculus of $f$ defined on the spectrum of $A$. Fundamental results about operator-monotone functions were collected in ([20], Section 2). Throughout this paper, $O M\left(\mathbb{R}^{+}\right)$is the set of operator-monotone functions from $\mathbb{R}^{+}=(0, \infty)$ to itself. The set $O M\left(\mathbb{R}^{+}\right)$and the set of finite (positive) Borel measures on $[0,1]$ are equipped with usual algebraic operations and pointwise orders. Recall that the $t$-weighted harmonic mean $!_{t}$ is defined by

$$
a !_{t} b=\left[(1-t) a^{-1}+t b^{-1}\right]^{-1}, \quad a, b>0 .
$$

This paper focuses on a one-to-one correspondence between four kind objects:

(i) monotone (Riemannian) metrics on $\mathcal{M}_{n}$

(ii) positive operator-monotone functions on $\mathbb{R}^{+}$

(iii) Morozowa-Chentsov functions on $\mathbb{R}^{+} \times \mathbb{R}^{+}$

(iv) finite (positive) Borel measures on $[0,1]$.

We show that there is a bijection between the finite Borel measures $\mu$ on $[0,1]$ and the positive operator-monotone functions $f$ via a canonical representation

$$
f(x)=\int_{0}^{1} 1 !_{t} x d \mu(t), \quad x \in \mathbb{R}^{+} .
$$

Moreover, the map $\mu \mapsto f$ is bijective, affine, and order-preserving. This means that the functions $x \mapsto 1 !_{t} x$ for $t \in[0,1]$ form building blocks for the set $O M\left(\mathbb{R}^{+}\right)$. This integral representation reflects some interesting information of operator-monotone functions. In fact, a function $f \in O M\left(\mathbb{R}^{+}\right)$is normalized if and only if its associated measure $\mu$ is a probability measure. We also show that an $f \in O M\left(\mathbb{R}^{+}\right)$is symmetric (in the sense that $f(x)=x f(1 / x)$ for all $x>0$ ) if and only if the corresponding measure $\mu$ is invariant under the function $t \mapsto 1-t$ on $[0,1]$. The normalized/symmetric conditions on $f \in O M\left(\mathbb{R}^{+}\right)$turn out to characterize such conditions for the associated monotone metrics and the associated Morozowa-Chentsov functions as well.

The canonical representation (1) also reflects the geometry of the set of (symmetric) normalized operator-monotone functions. More precisely, the extreme points of the convex set of such functions are obtained via the affinity of the map $\mu \mapsto f$. Furthermore, the representation (1) has benefits in decomposing positive operator-monotone functions as the sum of three explicit parts, namely, 
its singularly-discrete part, its absolutely-continuous part, and its singularly-continuous part. Such decomposition leads to a decomposition of the associated monotone metrics as well.

The rest of this paper is organized as follows. In Section 2, we recall fundamental results about monotone Riemannian metrics on the smooth manifold of invertible density matrices. Then, in Section 3, we establish an integral representation for positive operator-monotone functions with respect to a Borel measure on the unit interval. Moreover, we investigate some attractive properties from such representations. In Sections 4 and 5, we illustrate monotone metrics of type singularly-discrete and of type absolutely-continuous, respectively. Section 6 deals with decompositions of operator-monotone functions. We summarize the paper in Section 7.

\section{Monotone Riemannian Metrics on the Smooth Manifold of Invertible Density Matrices}

We denote the set of $(n \times n)$ complex matrices by $\mathcal{M}_{n}$. Recall that a density matrix is a positive semidefinite matrix with trace 1 . The set $\mathcal{D}_{n}$ of all $(n \times n)$ invertible density matrices is an open subset of the set of $(n \times n)$ Hermitian matrices. This is because the function $A \mapsto x^{*} A x$ is continuous for each $x \in \mathbb{C}^{n}$. Hence, the set $\mathcal{D}_{n}$ forms a smooth manifold.

A metric $K$ on $\mathcal{M}_{n}$ is a parametrized family $\left\{K_{D}\right\}_{D \in \mathcal{D}_{n}}$ of sesquilinear forms $K_{D}: \mathcal{M}_{n} \times \mathcal{M}_{n} \rightarrow \mathbb{C}$ such that

(i) $K_{D}$ is positive definite in the sense that $K_{D}(A, A) \geqslant 0$ for all $A \in \mathcal{M}_{n}$, and $K_{D}(A, A)=0$ if and only if $A=0$.

(ii) The map $D \mapsto K_{D}(A, A)$ is continuous for each $A \in \mathcal{M}_{n}$.

The metric $K$ is said to be monotone if for every $D \in \mathcal{D}_{n}, A \in \mathcal{M}_{n}$ and stochastic map $T: \mathcal{M}_{n} \rightarrow$ $\mathcal{M}_{n}$, we have

$$
K_{T(D)}(T(A), T(A)) \leqslant K_{D}(A, A)
$$

Here, recall that a linear map $T: \mathcal{M}_{n} \rightarrow \mathcal{M}_{n}$ is said to be stochastic if $T$ is completely positive and $T$ preserves invertible density matrices. It turns out that a differentiable monotone metric on $\mathcal{D}_{n}$ determines a Riemannian metric; see more information in [10].

Let $\langle\cdot, \cdot\rangle$ be the Hilbert-Schmidt inner product on $\mathcal{M}_{n}$, i.e.,

$$
\langle A, B\rangle=\operatorname{Tr}\left(A^{*} B\right) \quad \text { for any } A, B \in \mathcal{M}_{n} .
$$

For each $D \in \mathcal{D}_{n}$, let $L_{D}$ and $R_{D}$ be the left (right) multiplication operators from $\mathcal{M}_{n}$ to itself, i.e., $L_{D}: X \mapsto D X$ and $R_{D}: X \mapsto X D$. Then, $\left(L_{D}, R_{D}\right)$ is a pair of commuting invertible positive operators such that $L_{D}^{t}=L_{D^{t}}$ and $R_{D}^{t}=R_{D^{t}}$ for any $t \in \mathbb{R}$.

Morozowa and Chentsov [5] gave an explicit form of a monotone metric $K$. Indeed, for each $D \in \mathcal{D}_{n}$ and $A \in \mathcal{M}_{n}$, the value $K_{D}(A, A)$ appears in terms of the so-called associated Morozowa-Chentsov function, and we obtain $K_{D}(A, B)$ by means of polarization. Petz [6,7] improved this representation to the Hilbert-Schmidt inner product and operator-monotone function on $\mathbb{R}^{+}$ as follows:

Theorem 1. ([6,7]) There is a one-to-one correspondence between operator-monotone function $f: \mathbb{R}^{+} \rightarrow \mathbb{R}^{+}$ and monotone metric $K$ such that for any $D \in \mathcal{D}_{n}$ and $A, B \in \mathcal{M}_{n}$,

$$
K_{D}(A, B)=\left\langle A, \mathcal{K}_{D}^{-1}(B)\right\rangle=\operatorname{Tr} A^{*} g\left(L_{D}, R_{D}\right) B,
$$

where $\mathcal{K}_{D}=R_{D}^{\frac{1}{2}} f\left(L_{D} R_{D}^{-1}\right) R_{D}^{\frac{1}{2}}$ and $g$ is the associated Morozowa-Chentsov function defined by

$$
g(x, y)=\frac{1}{y f(x / y)} \quad \text { for any } x, y>0
$$


Here, $g\left(L_{D}, R_{D}\right)$ is computed by applying functional calculus on the pair of commuting operators $L_{D}$ and $R_{D}$.

\section{Characterizations of Positive Operator-Monotone Functions and Monotone Metrics}

In this section, we characterize operator-monotone functions from $\mathbb{R}^{+}$to $\mathbb{R}^{+}$in terms of finite positive Borel measures on the unit interval. These results give rise to characterizations of monotone metrics as well. The normalized/symmetric conditions for monotone metrics and operator-monotone functions are also considered.

For real sequences, we use the notation $x_{n} \uparrow x$ for the case that $\left(x_{n}\right)$ is an increasing sequence converging to $x$. The expression $x_{n} \downarrow x$ is used for the decreasing case.

Lemma 1. For given a finite (positive) Borel measure $\mu$ on $[0,1]$, the function

$$
f: \mathbb{R}^{+} \rightarrow \mathbb{R}^{+}, f(x)=\int_{0}^{1} 1 !_{t} x d \mu(t)
$$

is well-defined and continuous.

Proof. For each $x>0$, since $1 !_{t} x \leqslant \max (1, x)$ for any $t \in[0,1]$, we have

$$
\int_{0}^{1}\left|1 !_{t} x\right| d \mu(t) \leqslant \int_{0}^{1} \max (1, x) d \mu(t)=\max (1, x) \cdot \mu([0,1])<\infty .
$$

For each $x>0$, the positivity of the function $t \mapsto 1 !_{t} x$ implies that the resulting integral $f(x)$ is positive.

We shall show that $f$ is left and right continuous. First, note that the increasingness of function $x \mapsto 1 !_{t} x$ implies that $f$ is increasing. To show that $f$ is left continuous at a point $x>0$, let $\left(x_{n}\right)_{n=1}^{\infty}$ be a sequence in $\mathbb{R}^{+}$such that $x_{n} \uparrow x$. For convenience, put $\phi(t)=1 !_{t} x$ and $\phi_{n}(t)=1 !_{t} x_{n}$ for each $n \in \mathbb{N}$ and $t \in[0,1]$. Then $\left(\phi_{n}(t)\right)_{n=1}^{\infty}$ is a increasing sequence of positive real numbers such that $\phi_{n}(t) \rightarrow \phi(t)$ as $n \rightarrow \infty$ for each fixed $t$. It follows that the sequence $\left(f\left(x_{n}\right)\right)=\left(\int \phi_{n}\right)$ is increasing. Moreover, the monotone convergence theorem implies that

$$
f\left(x_{n}\right)=\int_{0}^{1} \phi_{n}(t) d \mu(t) \rightarrow \int_{0}^{1} \phi(t) d \mu(t)=f(x) .
$$

This means that $f\left(x_{n}\right) \uparrow f(x)$. Thus, $f$ is left continuous.

For the right continuity of $f$, let $x>0$ and consider a sequence $\left(x_{n}\right)_{n=1}^{\infty}$ in $\mathbb{R}^{+}$such that $x_{n} \downarrow x$. For convenience, put $\phi(t)=1 !_{t} x$ and $\phi_{n}(t)=1 !_{t} x_{n}$ for each $n \in \mathbb{N}$ and $t \in[0,1]$. Then, for each fixed $t$, the sequence $\left(\phi_{n}(t)\right)_{n=1}^{\infty}$ is a decreasing sequence in $\mathbb{R}^{+}$such that $\phi_{n}(t) \rightarrow \phi(t)$ as $n \rightarrow \infty$. It follows that the sequence $\left(f\left(x_{n}\right)\right)=\left(\int \phi_{n}\right)$ is decreasing. Note that the family $\left(\phi_{n}(t)\right)_{n=1}^{\infty}$ is bounded by an integrable function $\phi_{1}$. By the dominated convergence theorem, the sequence $\left(f\left(x_{n}\right)\right)$ converges to $f(x)$, hence, $f\left(x_{n}\right) \downarrow f(x)$. Therefore, $f$ is right continuous.

Lemma 2. A necessary and sufficient condition for a continuous function $f: \mathbb{R}^{+} \rightarrow \mathbb{R}^{+}$to be operator-monotone is that there is a unique finite Borel measure $v$ on $[0, \infty]$ such that

$$
f(x)=\int_{0}^{\infty} \frac{x(\lambda+1)}{x+\lambda} d v(\lambda), \quad x \in \mathbb{R}^{+} .
$$

Proof. See, e.g., ([20], Theorem 2.7.11). 
Theorem 2. There is a bijection between the set of finite Borel measure on $[0,1]$ and the set $O M\left(\mathbb{R}^{+}\right)$that sending a measure $\mu$ to an $f \in O M\left(\mathbb{R}^{+}\right)$satisfying the representation

$$
f(x)=\int_{0}^{1} 1 !_{t} x d \mu(t), \quad x \geqslant 0 .
$$

Moreover, the map $\mu \mapsto f$ is bijective, affine, and order-preserving.

Proof. The function $f$ in (5) is well-defined and continuous by Lemma 1 . To show that $f$ is operator-monotone, let us consider invertible operators $A$ and $B$ on a Hilbert space such that $A \leqslant B$. The monotonicity of weighted harmonic means and Bochner integrals implies that

$$
f(A)=\int_{0}^{1} I !_{t} A d \mu(t) \leqslant \int_{0}^{1} I !_{t} B d \mu(t)=f(B) .
$$

This means that the map $\mu \mapsto f$ is well-defined. For the injectivity of this map, let $\mu_{1}$ and $\mu_{2}$ be finite Borel measures on $[0,1]$ such that $f_{1}=f_{2}$ where

$$
f_{1}(x)=\int_{0}^{1} 1 !_{t} x d \mu_{1}(t), \quad f_{2}(x)=\int_{0}^{1} 1 !_{t} x d \mu_{2}(t), \quad x>0 .
$$

Then, for each $i=1,2$ and $x>0$, we get

$$
f_{i}(x)=\int_{0}^{\infty} \frac{x(\lambda+1)}{x+\lambda} d \mu_{i} \Psi(\lambda)
$$

where $\Psi:[0, \infty] \rightarrow[0,1], t \mapsto t /(t+1)$. Here, the measure $\mu_{i} \Psi$ is defined by $E \mapsto \mu_{i}(\Psi(E))$ for each Borel set $E$. Lemma 2 implies that $\mu_{1}=\mu_{2}$.

To show the surjectivity of this map, we consider $f \in O M\left(\mathbb{R}^{+}\right)$. By Lemma 2, there is a finite Borel measure $v$ on $[0, \infty]$ such that (4) holds. Define a finite Borel measure $\mu$ on $[0,1]$ by $\mu=v \Psi^{-1}$. A direct computation shows that

$$
f(x)=\int_{0}^{1} 1 !_{t} x d \mu(t), \quad x \geqslant 0 .
$$

Hence, the map $\mu \mapsto f$ is surjective. It is straightforward to show that this map is affine and order-preserving.

From Theorems 1 and 2, we get:

Corollary 1. There is a one-to-one correspondence between monotone metrics, Morozowa-Chentsov functions, and finite positive Borel measures on $[0,1]$ via the representations (2), (3), and (5).

The work [7] studied the normalized condition on a monotone metric $K_{D}(I, I)=\operatorname{Tr}\left(D^{-1}\right)$ in terms of the associated operator-monotone function. Recall that $f \in O M\left(\mathbb{R}^{+}\right)$is normalized if $f(1)=1$. The next result gives a complete characterization of normalized monotone metrics.

Corollary 2. Let $K$ be a monotone metric on $\mathcal{M}_{n}$ with the associated function $f \in O M\left(\mathbb{R}^{+}\right)$, the associated Morozowa-Chentsov function $g$, and the associated measure $\mu$ on $[0,1]$. Then the following statements are equivalent:

(i) $K_{D}(I, I)=\operatorname{Tr}\left(D^{-1}\right)$ for any $D \in \mathcal{D}_{n}$.

(ii) $f$ is normalized.

(iii) $g(x, x)=1 / x$ for any $x \in \mathbb{R}^{+}$.

(iv) $\mu$ is a probability measure. 
Thus, there is a one-to-one correspondence between normalized monotone metrics, normalized positive operator-monotone functions, and probability Borel measures on $[0,1]$ via the representations (2), (3), and (5).

Proof. The content of ([7], Corollary 6) indicates that the assertion (i) is equivalent to (ii). The assertion (ii) is clearly equivalent to (iii). The equivalence between (ii) and (iv) follows from the integral representation (5) in Theorem 2.

This corollary asserts that every normalized positive operator-monotone function can be regarded as an average of the special operator-monotone functions $x \mapsto 1 !_{t} x$ for $t \in[0,1]$.

Recall from [21] that if $f \in O M\left(\mathbb{R}^{+}\right)$, then the transpose of $f$, defined by $x \mapsto x f(1 / x)$ for any $x>0$, also belongs to $O M\left(\mathbb{R}^{+}\right)$. The function $f$ is said to be symmetric if it coincides with its transpose. We say that a Borel measure $\mu$ on $[0,1]$ is symmetric if $\mu \Theta=\mu$ where $\Theta:[0,1] \rightarrow[0,1], t \mapsto 1-t$. Recall also from [7] that a monotone metric $K$ is symmetric if $K_{D}(A, B)=K_{D}\left(B^{*}, A^{*}\right)$ for any $D \in \mathcal{D}_{n}$ and $A, B \in \mathcal{M}_{n}$.

The associated measure of transpose of $f \in O M\left(\mathbb{R}^{+}\right)$can be computed as follows.

Proposition 1. Let $f \in O M\left(\mathbb{R}^{+}\right)$be a function with associated measure $\mu$. Then the associated measure of the transpose of $f$ is given by $\mu \Theta$ where $\Theta:[0,1] \rightarrow[0,1], t \mapsto 1-t$.

Proof. It follows from the integral representation (5) of $f$ that

$$
x f\left(\frac{1}{x}\right)=x \int_{0}^{1} 1 !_{t} \frac{1}{x} d \mu(t)=\int_{0}^{1} x !_{t} 1 d \mu(t)=\int_{0}^{1} 1 !_{t} x d \mu \Theta(t) .
$$

By Theorem 2, the transpose of $f$ has $\mu \Theta$ as its associated measure.

The next result provides a complete characterization of symmetric monotone metrics.

Theorem 3. Let $K$ be a monotone metric on $\mathcal{M}_{n}$ with the associated function $f \in O M\left(\mathbb{R}^{+}\right)$, the associated Morozowa-Chentsov function $g$, and the associated measure $\mu$ on $[0,1]$. Then the following statements are equivalent:

(i) $\mathrm{K}$ is symmetric.

(ii) $f$ is symmetric.

(iii) $g(x, y)=g(y, x)$ for any $x, y \in \mathbb{R}^{+}$.

(iv) $\mu$ is symmetric.

Thus, there is a one-to-one correspondence between symmetric monotone metrics, symmetric positive operator-monotone functions, and symmetric Borel measures on $[0,1]$ via the representation (2) and the integral representation

$$
f(x)=\frac{1}{2} \int_{0}^{1}\left[\left(1 !_{t} x\right)+\left(x !_{t} 1\right)\right] d \mu(t), \quad x \in \mathbb{R}^{+} .
$$

Proof. The content of ([7], Theorem 7) indicates the equivalence between (i) and (ii). The latter condition is equivalent to (iii). From the formula (6) in Proposition 1 and the uniqueness of the associated measure (Theorem 2), we have that $f(x)=x / f(1 / x)$ if and only if $\mu=\mu \Theta$. Thus, the assertions (ii) and (iv) are equivalent. Theorem 1 establishes the correspondence between monotone metrics and operator-monotone functions via (2). From the canonical representation (5) and an observation that

$$
f(x)=\frac{x}{f(1 / x)}=\frac{1}{2}\left[f(x)+\frac{x}{f(1 / x)}\right],
$$

we can write the function $f$ in a symmetric form (7), relating $f$ to its associated measure. 
It follows from Corollaries 2 and 3 that there is a one-to-one correspondence between normalized symmetric positive operator-monotone functions and probability symmetric Borel measures on $[0,1]$ via the integral representation (7).

Note that the set of normalized (symmetric) operator-monotone functions on $\mathbb{R}^{+}$is a convex set. We denote the Dirac measure concentrated at a point $t$ by $\delta_{t}$. Now, the integral representation (5) also reflects the geometry of this set as follows.

\section{Corollary 3.}

(i) The (only) extreme points of the convex set of normalized positive operator-monotone functions are the functions $x \mapsto 1 !_{t} x$ where $t \in[0,1]$.

(ii) The functions $x \mapsto\left(1 !_{t} x+x !_{t} 1\right) / 2$ for $t \in[0,1]$ are extreme points of the convex set of normalized symmetric positive operator-monotone functions.

Proof. The assertions (i) and (ii) are consequences of the affinity of the map $\mu \mapsto f$ in Theorem 2 together with the following claims:

(1) The Dirac measures are the only extreme points of the convex set of probability Borel measures on $[0,1]$.

(2) The measures $\left(\delta_{t}+\delta_{1-t}\right) / 2$ for $t \in[0,1]$ are extreme points of the convex set of probability symmetric Borel measures on $[0,1]$.

To prove (1), note that the Dirac measures are extreme points of that set. Suppose there is a probability measure $\mu$ on $[0,1]$ which is an extreme point, but $\mu$ is not a Dirac measure. Then there is an $s \in[0,1]$ such that $0<\mu(\{s\})<1$. Define

$$
v=\frac{\mu-\mu(\{s\}) \delta_{s}}{1-\mu(\{s\})}
$$

We can verify that $v$ is a probability positive measure on $[0,1]$. It follows that

$$
\mu=\mu(\{s\}) \delta_{s}+(1-\mu(\{s\})) \nu
$$

i.e., $\mu$ is a non-trivial convex combination of two probability Borel measures. This contradicts the assumption that $\mu$ is an extreme point.

To prove (2), consider the measure $\left(\delta_{t}+\delta_{1-t}\right) / 2$ where $t \in[0,1]$. Suppose that there are a constant $\alpha \in(0,1)$ and probability measures $\mu_{1}, \mu_{2}$ on $[0,1]$, which are invariant under the function $\Theta$, such that

$$
\frac{1}{2}\left(\delta_{t}+\delta_{1-t}\right)=\alpha \mu_{1}+(1-\alpha) \mu_{2}
$$

For any $s \in[0,1]-\{t, 1-t\}$, we have

$$
0=\alpha \mu_{1}(\{s\})+(1-\alpha) \mu_{2}(\{s\})=\alpha \mu_{1}(\{1-s\})+(1-\alpha) \mu_{2}(\{1-s\}),
$$

so that $\mu_{1}(\{s\})=\mu_{1}(\{1-s\})=0$. Since $\mu_{1}$ is a probability measure, we get $\mu_{1}(\{t\})+\mu_{1}(\{1-t\})=1$. Since $\mu_{1}(\{t\})=\mu_{1}(\{1-t\})$, we have $\mu_{1}(\{t\})=\mu_{1}(\{1-t\})=1 / 2$. We now get $\mu_{1}=\left(\delta_{t}+\delta_{1-t}\right) / 2$ and, similarly, $\mu_{2}=\left(\delta_{t}+\delta_{1-t}\right) / 2$. Hence, the trivial combination is the only convex combination for $\left(\delta_{t}+\delta_{1-t}\right) / 2$, i.e., this measure is an extreme point of that set.

\section{Singularly-Discrete Monotone Metrics}

This section provides typical examples of "singularly-discrete" monotone metrics. 
Example 1. Consider the operator-monotone function $f(x)=1$. The associated Morozowa-Chentsov function is given by $c(x, y)=1 / y$. For each $D \in \mathcal{D}_{n}$, we have $\mathcal{K}_{D}=R_{D}$ and thus $\mathcal{K}_{D}^{-1}=R_{D^{-1}}$. Hence, its associated monotone metric is given by

$$
K_{D}(A, B)=\left\langle A, R_{D^{-1}}(B)\right\rangle=\operatorname{Tr}\left(D^{-1} A^{*} B\right) .
$$

The associated Borel measure on $[0,1]$ is given by the Dirac measure $\delta_{0}$.

Example 2. Consider the operator-monotone function $f(x)=x$. The associated Morozowa-Chentsov function is given by $c(x, y)=1 / x$. For each $D \in \mathcal{D}_{n}$, we have $\mathcal{K}_{D}=L_{D}$ and thus $\mathcal{K}_{D}^{-1}=L_{D^{-1}}$. Hence, its associated monotone metric is given by

$$
K_{D}(A, B)=\left\langle A, L_{D^{-1}}(B)\right\rangle=\operatorname{Tr}\left(A^{*} D^{-1} B\right) .
$$

Its associated Borel measure is given by the Dirac measure $\delta_{1}$.

Example 3. For each $t \in[0,1]$, the operator-monotone function $x \mapsto 1 !_{t} x$ corresponds to the Dirac measure $\delta_{t}$. By affinity of the map $\mu \mapsto f$, the measure $\sum_{i=1}^{n} a_{i} \delta_{t_{i}}$, where $t_{i} \in[0,1]$ and $a_{i} \geqslant 0$, is associated to the function $x \mapsto \sum_{i=1}^{n} a_{i}\left(1 !_{t_{i}} x\right)$.

Example 4. The smallest monotone metric is given by the Bures metric, introduced by Uhlmann [22]. This metric is represented by the Morozowa-Chentsov function

$$
c(x, y)=\frac{2}{x+y}
$$

which is associated to the operator-monotone function $f(x)=(1+x) / 2$. By Example 3, its associated measure of this metric is given by the probability measure $\left(\delta_{0}+\delta_{1}\right) / 2$.

More generally, let us consider the operator-monotone function $f(x)=1-\alpha+\alpha x$, where $\alpha \in[0,1]$. For each $D \in \mathcal{D}_{n}$, we have

$$
\mathcal{K}_{D}=R_{D}^{\frac{1}{2}} f\left(L_{D} R_{D}^{-1}\right) R_{D}^{\frac{1}{2}}=(1-\alpha) R_{D}+\alpha L_{D}
$$

so that $\mathcal{K}_{D}(X)=(1-\alpha) X D+\alpha D X$ for any $X \in \mathcal{M}_{n}$. Its associated measure is given by the probability measure $(1-\alpha) \delta_{0}+\alpha \delta_{1}$.

Example 5. The largest monotone metric is the metric represented by the Morozowa-Chentsov function

$$
c(x, y)=\frac{x+y}{2 x y} .
$$

This metric is associated to the operator-monotone function $f(x)=2 x /(x+1)$. It follows from Example 3 that its associated measure of this metric is $\delta_{1 / 2}$.

More generally, let us consider the operator-monotone function

$$
f(x)=\frac{x}{\alpha+(1-\alpha) x}
$$

where $\alpha \in[0,1]$. For each $D \in \mathcal{D}_{n}$, a direct computation reveals that

$$
\mathcal{K}_{D}=\left[(1-\alpha) R_{D^{-1}}+\alpha L_{D^{-1}}\right]^{-1} .
$$

Its associated measure is given by the probability measure $\delta_{\alpha}$. 
Remark 1. In Theorem 2, the map $f \mapsto \mu$ is not order-preserving in general. Consider $f(x)=2 x /(1+x)$ and $g(x)=(1+x) / 2$. Then $\mu_{f}=\delta_{1 / 2}$ and $\mu_{g}=\left(\delta_{0}+\delta_{1}\right) / 2$. We have $f \leqslant g$ but $\mu_{f} \nless \mu_{g}$.

\section{Absolutely-Continuous Monotone Metrics}

In this section, we illustrate the one-to-one correspondence between positive operator-monotone functions, absolutely-continuous Borel measures, and certain type of monotone metrics. We call such metrics absolutely-continuous monotone metrics. Such functions will be typical examples of absolutely-continuous type in the next section.

Example 6. Consider the operator-monotone function $x \mapsto \log (1+x)$. Using improper integration, we have

$$
\log (1+x)=\int_{1}^{\infty} \frac{x}{\lambda(x+\lambda)} d \lambda=\int_{1 / 2}^{1}\left(1 !_{t} x\right) \frac{(1-t)^{2}}{t} d\left(\frac{t}{1-t}\right)=\int_{0}^{1}\left(1 !_{t} x\right) h(t) d t
$$

where $h(t)=(1 / t) \chi_{[1 / 2,1]}(t)$. Here, $\chi_{[1 / 2,1]}$ denotes the characteristic function on the set $[1 / 2,1]$. Thus, its associated measure is given by the absolutely-continuous measure having $h$ as its density function. Hence, the function $\log (1+x)$ gives rise to a monotone metric.

Example 7. For each $0<\alpha<1$, consider the Morozowa-Chentsov function $c(x, y)=x^{-\alpha} y^{\alpha-1}$. This function is associated to the operator-monotone function $f(x)=x^{\alpha}$. For each $D \in \mathcal{D}_{n}$, we have

$$
\begin{aligned}
\mathcal{K}_{D} & =R_{D}^{\frac{1}{2}}\left(R_{D}^{-\frac{1}{2}} L_{D} R_{D}^{-\frac{1}{2}}\right)^{\alpha} R_{D}^{\frac{1}{2}}=R_{D}^{\frac{1}{2}} R_{D}^{-\frac{\alpha}{2}} L_{D}^{\alpha} R_{D}^{-\frac{\alpha}{2}} R_{D}^{\frac{1}{2}} \\
& =R_{D^{\frac{1}{2}}} R_{D^{-\frac{\alpha}{2}}} L_{D^{\alpha}} R_{D^{-\frac{\alpha}{2}}} R_{D^{\frac{1}{2}}}=R_{D^{\frac{1-\alpha}{2}}} L_{D^{\alpha}} R_{D^{\frac{1-\alpha}{2}}}=L_{D^{\alpha}} R_{D^{1-\alpha}}
\end{aligned}
$$

Thus, the associated monotone metric is given by

$$
K_{D}(A, B)=\left\langle A, \mathcal{K}_{D}^{-1}(B)\right\rangle=\left\langle A, D^{-\alpha} B D^{\alpha-1}\right\rangle=\operatorname{Tr}\left(A^{*} D^{-\alpha} B D^{\alpha-1}\right) .
$$

To compute its associated measure, we recall a standard result from contour integrations:

$$
x^{\alpha}=\int_{0}^{\infty} \frac{x t^{\alpha-1}}{x+t} \cdot \frac{\sin \alpha \pi}{\pi} d t
$$

Denoting $\Psi(t)=t /(1-t)$, we have

$$
x^{\alpha}=\int_{0}^{1}\left(1 !_{t} x\right) \Psi(t)^{\alpha-1} \cdot \frac{1}{\Psi(t)+1} \cdot \frac{\sin \alpha \pi}{\pi} d \Psi(t)=\int_{0}^{1}\left(1 !_{t} x\right) d \mu(t),
$$

where the associated measure $\mu$ is given by

$$
d \mu(t)=\frac{\sin \alpha \pi}{\pi} \cdot \frac{1}{t^{1-\alpha}(1-t)^{\alpha}} d t .
$$

Example 8. The Kubo-Mori-Bogoliubov metric with Morozowa-Chentsov function

$$
c(x, y)=\frac{\log x-\log y}{x-y}
$$

is associated to the operator-monotone function $f(x)=(x-1) / \log x$. Using Example 7 and Fubini's theorem, we have

$$
f(x)=\int_{0}^{1} x^{\lambda} d \lambda=\int_{0}^{1} \int_{0}^{1} \frac{\sin \lambda \pi}{\pi} \cdot \frac{1 !_{t} x}{t^{1-\lambda}(1-t)^{\lambda}} d t d \lambda=\int_{0}^{1}\left(1 !_{t} x\right) \int_{0}^{1} \frac{\sin \lambda \pi}{\pi t^{1-\lambda}(1-t)^{\lambda}} d \lambda d t .
$$


This means that the associated measure of $f$ is given by $d \mu(t)=h(t) d t$, where

$$
h(t)=\int_{0}^{1} \frac{\sin \lambda \pi}{\pi t^{1-\lambda}(1-t)^{\lambda}} d \lambda .
$$

Using integration by parts twice, we have

$$
\int \frac{\sin \pi \lambda}{t^{1-\lambda}(1-t)^{\lambda}} d \lambda=\frac{1}{\pi^{2}+\log ^{2}\left(\frac{t}{1-t}\right)} \cdot\left[\frac{-\pi \cos \pi \lambda}{t^{1-\lambda}(1-t)^{\lambda}}+\log \left(\frac{t}{1-t}\right) \cdot \frac{\sin \pi \lambda}{t^{1-\lambda}(1-t)^{\lambda}}\right]+C .
$$

Hence,

$$
h(t)=\frac{1}{\pi} \int_{0}^{1} \frac{\sin \lambda \pi}{t^{1-\lambda}(1-t)^{\lambda}} d \lambda=\frac{1}{t(1-t)\left(\pi^{2}+\log ^{2}\left(\frac{t}{1-t}\right)\right)} .
$$

Example 9. Consider the dual $1 / f(1 / x)$ of the function $f$ in Example 8. We have the integral representation

$$
\frac{1}{f(1 / x)}=\frac{x}{x-1} \log x=\int_{0}^{1} 1 !_{t} x d t
$$

that is, this function corresponds to the Lebesgue measure.

\section{Explicit Descriptions of Positive Operator-Monotone Functions}

In this section, we give an explicit description of arbitrary operator-monotone functions on $\mathbb{R}^{+}$by decomposing them into typical concrete ones we have encountered in Sections 4 and 5. It is important to note that the one-to-one correspondences (2) and (5) are both affine. Thus, if we can decompose an operator-monotone function, then it gives rise to a decomposition of the associated monotone metric as well. We also investigate such decomposition when such functions are normalized or symmetric. For this section, we denote Lebesgue measure by $m$.

Theorem 4. For each $f \in O M\left(\mathbb{R}^{+}\right)$, there is a unique triple $\left(f_{a c}, f_{s c}, f_{s d}\right)$ of operator-monotone functions on $\mathbb{R}^{+}$such that $f=f_{a c}+f_{s c}+f_{s d}$ and

(i) there are a countable set $D \subseteq[0,1]$ and a summable family $\left\{a_{t}\right\}_{t \in D} \subseteq \mathbb{R}^{+}$such that for each $x \in \mathbb{R}^{+}$

$$
f_{s d}(x)=\sum_{\lambda \in D} a_{t}\left(1 !_{t} x\right)
$$

(ii) there is a (unique m-a.e.) integrable function $h:[0,1] \rightarrow \mathbb{R}^{+}$such that

$$
f_{a c}(x)=\int_{0}^{1} h(t)\left(1 !_{t} x\right) d m(t), \quad x \in \mathbb{R}^{+} ;
$$

(iii) its associated measure of $f_{\text {sc }}$ is continuous and mutually singular to $m$.

Moreover, the associated measure of $f_{s d}$ is given by $\sum_{t \in D} a_{t} \delta_{t}$.

Proof. Let $\mu$ be the associated measure of $f$. By a standard result in measure theory (e.g., [23]), there is a unique triple $\left(\mu_{a c}, \mu_{s c}, \mu_{s d}\right)$ of finite Borel measures on $[0,1]$ such that $\mu=\mu_{a c}+\mu_{s c}+\mu_{s d}$ where

(I) $\mu_{s d}$ is a discrete measure

(II) $\mu_{a c}$ is absolutely continuous with respect to $m$

(III) $\mu_{s c}$ is a continuous measure mutually singular to $m$. 
Define

$$
f_{a c}(x)=\int_{0}^{1} 1 !_{t} x d \mu_{a c}, \quad f_{s d}(x)=\int_{0}^{1} 1 !_{t} x d \mu_{s d}, \quad f_{s c}(x)=\int_{0}^{1} 1 !_{t} x d \mu_{s c} .
$$

Then $f_{a c}, f_{s d}, f_{s c} \in O M\left(\mathbb{R}^{+}\right)$and $f=f_{a c}+f_{s d}+f_{s c}$. The condition (I) means precisely that there are a countable set $D \subseteq[0,1]$ and a family $\left\{a_{\lambda}\right\}_{\lambda \in D}$ in $\mathbb{R}^{+}$such that $\sum_{\lambda \in D} a_{\lambda}<\infty$ and $\mu_{s d}=\sum_{t \in D} a_{t} \delta_{t}$. Hence, we arrive at the formula (8). Note that this series converges since

$$
\sum_{t \in D} a_{t}\left(1 !_{t} x\right) \leqslant \sum_{t \in D} a_{t} \max (1, x)<\infty, \quad x \in \mathbb{R}^{+} .
$$

The condition (II) means precisely the condition (ii) by Radon-Nikodym theorem. The uniqueness of $\left(f_{a c}, f_{s c}, f_{s d}\right)$ follows from the uniqueness of $\left(\mu_{a c}, \mu_{s c}, \mu_{s d}\right)$ and the correspondence between operator-monotone functions and measures. The measure $\sum_{t \in D} a_{t} \delta_{t}$ is associated to $f_{s d}$ since the associated measure of $x \mapsto 1 !_{t} x$ is $\delta_{t}$ for each $t \in[0,1]$ by Example 3 .

Theorem 4 asserts that every $f \in O M\left(\mathbb{R}^{+}\right)$consists of three parts. The singularly-discrete part $f_{\text {sd }}$ is a countable sum of $x \mapsto 1 !_{t} x$ for $t \in[0,1]$, given by (8). Such type of functions include the straight lines with positive slopes, the constant functions, the multiple functions $x \mapsto k x$, and the examples in Section 4 . The absolutely-continuous part $f_{a c}$ arises explicitly as an integral with respect to Lebesgue measure given by (9). Typical examples of such functions are already provided in Section 5 . The singularly-continuous part $f_{s c}$ admits an integral representation with respect to a continuous measure mutually singular to Lebesgue measure.

Proposition 2. The operator-monotone function $f_{a c}$ defined by (9) is normalized if and only if the average of the density function $h$ is 1 , i.e., $\int_{0}^{1} h(t) d t=1$. This function is symmetric if and only if $h \circ \Theta=h$.

Proof. It follows from Corollary 2 and Theorem 3.

We say that a density function $h:[0,1] \rightarrow \mathbb{R}^{+}$is symmetric if $h \circ \Theta=h$. Next, we decompose a normalized operator-monotone function as a convex combination of normalized operator-monotone functions.

Corollary 4. Let $f \in O M\left(\mathbb{R}^{+}\right)$be normalized. Then there are

- a unique triple $\left(\widetilde{f}_{a c}, \widetilde{f}_{s c}, \widetilde{f}_{s d}\right)$ of normalized operator-monotone functions or zero functions,

- a unique triple $\left(k_{a c}, k_{s c}, k_{s d}\right)$ of real numbers in $[0,1]$

such that

$$
f=k_{a c} \widetilde{f}_{a c}+k_{s c} \widetilde{f}_{s c}+k_{s d} \widetilde{f}_{s d}, \quad k_{a c}+k_{s c}+k_{s d}=1,
$$

and

(i) there are a countable set $D \subseteq[0,1]$ and a family $\left\{a_{t}\right\}_{t \in D} \subseteq[0,1]$ such that $\sum_{t \in D} a_{t}=1$ and $f_{\text {sd }}(x)=$ $\sum_{\lambda \in D} a_{t}\left(1 !_{t} x\right)$ for each $x \in \mathbb{R}^{+}$;

(ii) there is a (unique m-a.e.) integrable function $h:[0,1] \rightarrow \mathbb{R}^{+}$with average 1 such that $f_{a c}(x)=$ $\int_{0}^{1} h(t)\left(1 !_{t} x\right) d m(t)$ for $x \in \mathbb{R}^{+}$;

(iii) its associated measure of $f_{s c}$ is continuous and mutually singular to $\mathrm{m}$. 
Proof. Let $\mu$ be the associated probability measure of $f=f_{a c}+f_{s d}+f_{s c}$ and write $\mu=\mu_{a c}+\mu_{s d}+\mu_{s c}$. Suppose that $\mu_{a c}, \mu_{s d}$ and $\mu_{s c}$ are nonzero. Set

$$
\begin{aligned}
& \tilde{\mu}_{a c}=\frac{\mu_{a c}}{\mu_{a c}([0,1])}, \quad \tilde{\mu}_{s d}=\frac{\mu_{s d}}{\mu_{s d}([0,1])}, \quad \tilde{\mu}_{s c}=\frac{\mu_{s c}}{\mu_{s c}([0,1])}, \\
& k_{a c}=\mu_{a c}([0,1]), \quad k_{s d}=\mu_{s d}([0,1]), \quad k_{s c}=\mu_{s c}([0,1]) .
\end{aligned}
$$

Define $\widetilde{f}_{a c}, \widetilde{f}_{s d}, \widetilde{f}_{s c}$ to be the functions corresponding to the measures $\widetilde{\mu}_{a c}, \widetilde{\mu}_{s d}, \widetilde{\mu}_{s c}$, respectively. Now, let us apply Theorem 4 and Proposition 2.

We can decompose a symmetric operator-monotone function as a nonnegative linear combination of symmetric operator-monotone functions as follows:

Corollary 5. Let $f \in O M\left(\mathbb{R}^{+}\right)$be symmetric. Then there is a unique triple $\left(f_{a c}, f_{s c}, f_{s d}\right)$ of symmetric operator-monotone functions such that $f=f_{a c}+f_{s c}+f_{s d}$ and

(i) there are a countable set $D \subseteq[0,1]$ and a summable family $\left\{a_{t}\right\}_{t \in D} \subseteq \mathbb{R}^{+}$such that $a_{t}=a_{1-t}$ for all $t \in D$, and $f_{s d}(x)=\sum_{t \in D} a_{t}\left(1 !_{t} x\right)$ for each $x \in \mathbb{R}^{+} ;$

(ii) there is a (unique m-a.e.) symmetric integrable function $h:[0,1] \rightarrow \mathbb{R}^{+}$such that

$$
f_{a c}(x)=\frac{1}{2} \int_{0}^{1} h(t)\left(1 !_{t} x+x !_{t} 1\right) d m(t), \quad x \geqslant 0 ;
$$

(iii) its associated measure of $f_{s c}$ is continuous and mutually singular to $\mathrm{m}$.

Proof. Let $\mu$ be the associated measure of $f$. Decompose $\mu=\mu_{a c}+\mu_{s d}+\mu_{s c}$ where $\mu_{a c} \ll m$, the measure $\mu_{s d}$ is discrete, $\mu_{s c}$ is continuous, and $\mu_{s c} \perp m$. Then $\mu \Theta=\mu_{a c} \Theta+\mu_{s d} \Theta+\mu_{s c} \Theta$ where $\Theta:[0,1] \rightarrow[0,1], t \mapsto 1-t$. It is straightforward to verify that $\mu_{a c} \Theta \ll m$, the measure $\mu_{s d} \Theta$ is discrete, $\mu_{s c} \Theta$ is continuous, and $\mu_{s c} \Theta \perp m$. By Theorem 3, $\mu \Theta=\mu$. The uniqueness of measure decomposition implies that $\mu_{a c} \Theta=\mu_{a c}, \mu_{s d} \Theta=\mu_{s d}$ and $\mu_{s c} \Theta=\mu_{s c}$. Again, by Theorem $3 f_{a c}, f_{s d}$, and $f_{s c}$ are symmetric operator-monotone functions. Finally, let us apply Theorem 4 and Proposition 2.

A decomposition of any normalized symmetric operator-monotone function as a convex combination of such ones is also obtained by the normalizing process as in the proof of Corollary 4 .

Example 10. Recall that the Wigner-Yanase metric is represented by the Morozowa-Chentsov function

$$
c(x, y)=\frac{4}{(\sqrt{x}+\sqrt{y})^{2}} .
$$

Its associated operator-monotone function is given by

$$
f(x)=\frac{1}{4}(x+2 \sqrt{x}+1)
$$

We see that this function is a convex combination of two singularly-discrete operator-monotone functions and an absolutely-continuous one. By Example 7 and Theorem 2, its associated measure on the unit interval is

$$
\mu=\frac{1}{4}\left(\delta_{1}+2 v+\delta_{0}\right)
$$

where $d v(t)=(\pi \sqrt{t(1-t)})^{-1} d t$. 


\section{Conclusions}

There are strongly connections between positive operator-monotone functions on the positive reals, monotone (Riemannian) metrics, Morozowa-Chentsov functions, and finite Borel measures on the unit interval. Indeed, there are one-to-one correspondences between the four kind objects. It follows that certain properties (e.g., symmetry, normalization) of monotone metrics can be investigated through the associated properties of the other objects. Moreover, we can decompose the operator-monotone functions (thus, the other objects) into three parts, namely, its singularly-discrete part, its absolutely-continuous part, and its singularly-continuous part. Concrete monotone metrics in quantum Fisher information theory are illustrated with the associated operator-monotone functions, the associated Morozowa-Chentsov functions, and the associated measures.

Author Contributions: Project administration, P.C.; Writing-original draft, P.C.; Writing-review and editing, S.V.S. All authors contributed equally and significantly in writing this article. All authors read and approved the final manuscript.

Funding: This research was supported by King Mongkut's Institute of Technology Ladkrabang Research Fund, grant no. KREF046201.

Acknowledgments: The authors would like to thank referees for useful advices to make a better presentation of the paper.

Conflicts of Interest: The authors declare no conflict of interest.

\section{References}

1. Petz, D.; Ghinea, C. Introduction to Quantum Fisher Information. In Quantum Probability and Related Topics; Rebolledo, R., Orszag, M., Eds.; World Scientific: Singapore, 2011; pp. 261-281.

2. Wigner, E.P.; Yanase, M.M. Information contents of distributions. Proc. Natl. Acad. Sci. USA 1963, 49, 910-918. [CrossRef] [PubMed]

3. Lieb, E.H. Convex trace functions and the Wigner-Yanase-Dyson conjecture. Adv. Math. 1973, 11, $267-288$. [CrossRef]

4. Chentsov, N.N. Staistical decision rules and optimal inferences. Transl. Math. Monogr. 1982, 53, 1-499.

5. Morozowa, E.A.; Chentsov, N.N. Markov invariant geometry on state manifolds. J Soviet Math. 1991, 56, 2648-2669. (Translated form Russian) [CrossRef]

6. Petz, D. Geometry of canonical correlation on the state space of a quantum system. J. Math. Phys. 1994, 35, 780-795. [CrossRef]

7. Petz, D. Monotone metrics on matrix spaces. Linear Algebra Appl. 1996, 244, 81-96. [CrossRef]

8. Hansen, F. Characterizations of symmetric monotone metrics on the state space of quantum systems. Quant. Inf. Comput. 2006, 6, 597-605.

9. Hansen, F. Metric adjusted skew information. Proc. Natl. Acad. Sci. USA 2008, 105, 9909-9916. [CrossRef] [PubMed]

10. Hiai, F.; Petz, D. Riemannian metrics on positive definite matrices related to means. Linear Algebra Appl. 2009, 430, 3105-3130. [CrossRef]

11. Ando, T.; Hiai, F. Operator log-convex functions and operator means. Math. Ann. 2010, 350, 611-630. [CrossRef]

12. Hansen, F. Convexity of quantum $\chi^{2}$-divergence. Proc. Natl. Acad. Sci. USA 2011, 108, 10078-10080. [CrossRef] [PubMed]

13. Gibilisco, P.; Isola, T. Wigner-Yanase information on quantum state space: The geometric approach. J. Math. Phys. 2003, 44, 3752-3762. [CrossRef]

14. Hansen, F. WYD-like Skew Information Measures. J. Stat. Phys. 2013, 151, 974-979. [CrossRef]

15. Fujii, J.; Kamei, E. Relative operator entropy in noncommutative information theory. Math. Japon. 1989, 34, 341-348.

16. Chen, Z. Wigner-Yanase skew information as tests for entanglement. Phys. Rev. A 2005, 71, 1-5. [CrossRef]

17. Gibilisco, P.; Hiai, F.; Petz, D. Quantum covariance, quantum Fisher information and the uncertainty principle. IEEE Trans. Inform. Theory 2009, 55, 439-443. [CrossRef] 
18. Anderson, W.N.; Trapp, G.E. A class of monotone operator functions related to electrical network theory. Linear Algebra Appl. 1975, 15, 53-67. [CrossRef]

19. Tonchev, N.S. Monotone Riemannian metrics and dynamic structure factor in condensed matter physics. J. Math. Phys. 2016, 57, 071903. [CrossRef]

20. Hiai, F. Matrix analysis: matrix monotone functions, matrix means, and majorizations. Interdiscip. Inform. Sci. 2010, 16, 139-248. [CrossRef]

21. Hansen, F.; Pedersen, G.K. Jensen's inequality for operators and Löwner's theorem. Math. Ann. 1982, 258, 229-241. [CrossRef]

22. Uhlmann, A. The metric of Bures and the geometric phase. In Groups and Related Topics; Kluwer Academic Publishers: Dordrecht, The Netherlands, 1992.

23. Folland, G.B. Real Analysis: Modern Techniques and Their Applications; John Wiley \& Sons: New York, NY, USA, 1999.

(C) 2019 by the authors. Licensee MDPI, Basel, Switzerland. This article is an open access article distributed under the terms and conditions of the Creative Commons Attribution (CC BY) license (http:/ / creativecommons.org/licenses/by/4.0/). 